\title{
Identification of Viruses Infecting Cigar and Flue- cured Tobacco Using Next Generation Sequencing and Establishment of Multiplex RT-PCR
}

\section{Tao Zhou}

Shenyang Agricultural University

\section{Shidong Zhou}

Shenyang Agricultural University

\section{Yong Chen}

Sichuan tobacco company Deyang city company

Jun Wang

Sichuan tobacco company Deyang city company

Ruina Zhang

Sichuan tobacco company Deyang city company

\section{Huan Xiang}

Sichuan tobacco company Deyang city company

\section{Zihao Xia}

Shenyang Agricultural University

\section{Mengnan An}

Shenyang Agricultural University

Xiuxiang Zhao ( $\square$ zhaoxx0772@syau.edu.cn )

Shenyang Agricultural University

\section{Yuanhua Wu}

Shenyang Agricultural University

\section{Research Article}

Keywords: Plant virus, Cigar tobacco, Synergistic infection, Deep sequencing, Multiplex RT-PCR

Posted Date: October 1st, 2021

DOI: https://doi.org/10.21203/rs.3.rs-202195/v2

License: (c) (i) This work is licensed under a Creative Commons Attribution 4.0 International License.

Read Full License 


\section{Abstract}

Early, precise and simultaneous identification of the plant viruses is of great significance on preventing the spread of the viruses as well as reducing losses on agricultural yield. In this study, identification of plant viruses from symptomatic samples collected from cigar tobacco planting area in Deyang and fluecured tobacco planting area in Luzhou city of Sichuan Province China was conducted by the deep sequencing of small RNAs (sRNAs) through an Illumina sequencing platform and plant virus specific contigs were generated based on the virus derived siRNA sequences. Additionally, sequence alignment and phylogenetic analysis was performed to determine the species or strains of these viruses. A total of 27930450, 21537662 and 28194021 clean reads were generated from three pooled samples with a total of 105 contigs being mapped to the closest plant viruses with the length range from 34 1720 nt. The results indicated that the major viruses were potato virus Y (PVY), Chilli veinal mottle virus (ChiVMV), tobacco vein banding mosaic virus (TVBMV), tobacco mosaic virus (TMV) and cucumber mosaic virus (CMV). Subsequently, a fast and sensitive multiplex reverse transcription polymerase chain reaction (RTPCR) assay was developed for the simultaneous detection of the most frequent RNA viruses infecting cigar and flue-cured tobacco in Sichuan. These results provide theoretical basis and convenient methods for rapid detection and control of viruses on cigar and flue-cured tobacco.

\section{Introduction}

Crop losses due to plant viral infection poses serious threat to food sustenance and is of great concern worldwide (Scholthof et al., 2011). Plant viruses induce symptoms such as mosaic, mottling, yellowing, ring spots, venial necrosis, stunting, shoestring and deformation of the hosts, which may result in significant losses or quality reduction (Scholthof et al., 2011; Wang et al, 2019). Synergistic infection by multiple species of viruses often cause more serious symptoms and damages of the plants (Moreno and López-Moya 2020; Xia et al., 2016). Therefore, early, precise and simultaneous identification of the plant viruses is of great significance on preventing the spread of the viruses as well as reducing losses on agricultural yield. The multiplex RT-PCR assay is a widely-used molecular diagnostic technique used for the amplification of multiple target sequences of different pathogens in a single PCR experiment (Bi et al., 2019; Zhou et al., 2017).

RNA silencing in plant is one of the major defense systems to combat viruses, in which the exogenous viral double stranded RNA (dsRNA) is perceived and cut by DICER-like (DCL) proteins to produce primary virus-derived small interfering RNAs (vsiRNAs) and amplified by RNA-dependent RNA polymerases (RDRs) to generate secondary vsiRNAs of between 21 to $25 \mathrm{nt}$ (Zhang et al., 2015; Ding and Lu. 2011). Global investigation of the vsiRNAs using next-generation sequencing (NGS) approaches are effective on the identification of the reported or novel viruses (Wu et al., 2012; Vivek et al., 2020; Kreuze et al., 2009).

Plants belong to Solanaceae, especially tobacco (Nicotiana tabacum) is a natural host for a large number of plant viruses (Akinyemi et al.2016; Sun et al. 2016). In China, more than 25 plant viruses have been identified from tobacco. TMV is the most well investigated plant virus that cause continuous losses to 
tobacco production (Shaw 1999). In addition to TMV, various viruses belong to the genus Potyvirus including PVY, ChiVMV, TVBMV and tobacco etch virus (TEV) can also independently or synergistically infect tobacco plants and significantly decrease their quality (Akinyemi et al., 2016; Jiao et al., 2020; Dai et al., 2012). PVY is a prevalent virus with wide host range that can induce vein necrotic or leaf mosaic symptoms in tobacco based on their strains (Cuevas et al., 2012; Faurez et al., 2012; Tian and Valkonen, 2015). Infection of TVBMV can cause bands of dark green tissue along the veins as well as small white to brown spots on the leaves (Geng et al., 2017). Additionally, ChiVMV induce leaf punctate necrosis with round bright yellow spots and deformation symptoms on tobacco(Jiao et al., 2020; Rao et al., 2020; Yang et al., 2018).

Sichuan province is one of the main regions for tobacco production in China and is located in the subtropics area, with abundant species of vegetation, plant viruses as well as their vector insects such as aphids and Bemisia tabaci. Especially in cigar tobacco growing areas of Deyang city, tobacco is mixed planted with crops such as peppers and garlic, which significantly increases the risks of transmission of different virus species. In this study, we used NGS technique of small RNA sequencing to identify viruses from symptomatic tobacco plants in farm fields of Deyang and Luzhou of Sichuan province. The results indicated that major viruses are PVY, ChiVMV, TVBMV, TMV and CMV. We also performed sequence alignment to reveal the phylogenetic relationships of the viruses. Additionally, we established a multiplex RT-PCR for simultaneous detection of these viruses, which is applicable for the rapid virus identification and control.

\section{Materials And Methods}

\section{Collection and preparation of samples}

In the summer of 2019, more than 80 leaf samples with mosaic, mottling, yellowing, stunting or deformation symptoms of Nicotiana tabacum variety cigar (cigar tobacco) with distinct viral symptoms were collected from Deyang city in Sichuan Province of China (Longitude: $104^{\circ} 10^{\prime} 3$ ", latitude: $31^{\circ} 7^{\prime} 36^{\prime \prime}$ ). In addition, the viral disease symptomatic leaves of Nicotiana tabacum variety Yunyan85 and Yunyan87 were collected from Luzhou city in Sichuan province in China (Longitude: $105^{\circ} 38^{\prime} 28^{\prime \prime}$, latitude: $\left.28^{\circ} 7^{\prime} 33^{\prime \prime}\right)$. The collected cigar tobacco and flue-cured tobacco leaf samples were immediately frozen in liquid nitrogen and preserved at $-80^{\circ} \mathrm{C}$ for extraction and some were used for plant inoculation and highthroughput sequencing.

\section{Small RNA library construction, sequencing and bioinformatics analysis}

Total RNA were extracted from the collected cigar tobacco and flue-cured tobacco leaf samples using a mirVana $^{\text {TM }}$ miRNA Isolation Kit $\$ Cat \#. AM1561, Austin TX, US囚according to the manufacturers guidelines and subjected to detection using Agilent 2100 Bioanalyzer (Agilent technologies Santa Clara, USA). The high-throughput sequencing was performed on an Illumina HiSeq-2000 sequencing plat form (Shanghai Biotechnology Corporation, Shanghai, China). Briefly, for the symptomatic cigar tobacco and flue-cured

tobacco leaf samples small RNA libraries, the low quality reads and 5'- and 3'-adaptor contaminants were 
clipped from raw reads, and then clean reads ranging from 18- to 28-nt in length, which can be mapped to the genome sequences of plant viruses were extracted for analyses. The contigs with the length range from 34 1720 nt were assembled based on the virus derived siRNA sequences using the scaffolding contig algorithm (CLC Genomics Workbench version:6.0.4) (Su et al., 2011) and Cap3 EST splicing software. Sequences of the contigs were used to query the GenBank databases using the Basic Local Alignment Search Tool (BLAST) and mapped to the closest plant viral genome. Final hits with nucleic acid coverage $\geq 90 \%$ and identity $\geq 90 \%$ of the viruses were identified and aligned to determine their positions.

\section{Virus amplification and sequencing validation}

Total RNA was extracted from the above preserved leaves using Eastep Super Total RNA Extraction Kit (Promega, Shanghai, China). Total RNA was used as a template for RT-PCR, and five pairs of specific primers were designed based on alignment of the contigs and the genomic sequences of the PVY (no, MN734254.1), ChiVMV (no, KC711055.1), TVBMV (no, EF219408.1) TMV (no, HE818449.1) and CMV (no, NC-001440.1), respectively, from the GeneBank. PVY-5719F/PVY-6263R, ChiVMV-2441F/ChiVMV3453R, TVBMV-1071F/TVBMV-2425R, TMV-70F/TMV-2061R and CMV-1257F/CMV-1913R were shown in Table 1. The amplified PCR products were purified using a SanPrep Column PCR Product Purification kit (Sangon Biotech, Shanghai, China) and cloned into the pEASY-T1 vector (TransGen Biotech, Beijing, China).

\section{Phylogenetic analysis}

The nucleotide sequences of PVY Vpg, ChiVMV P3, TVBMV HC-pro, the MET domain of TMV p126 together with CP of CMV were amplified using PVY-5719F/PVY-6263R, ChiVMV-2441F/ChiVMV-3453R, TVBMV-1071F/TVBMV-2425R, TMV-70F/TMV-2061R and CMV-1257F/CMV-1913R, respectively, and compared with other related virus isolates whose sequences were deposited in GenBank. Homologous genes were searched using BLAST program in NCBI website. Sequences of the same virus species were aligned by Clustal W version 1.6 and the phylogenetic analysis was performed by using MEGA7 software (Kumar et al., 2016) with the maximum likelihood algorithm and statistical test with 1000 replicates to construct phylogenetic tree of those viruses.

\section{Multiplex RT-PCR analysis}

At least 20 different strains of each virus originating from different geographical regions were retrieved from the NCBI database and used for multiple sequence alignment. The sequence identity was at least $98 \%$ for each virus and conserved regions were chosen for primer design. Due to the frequent occurrence of CMV in tobacco planting field, we also added CMV detection primers in multiplex RT-PCR analysis. Each primer pair was designed to amplify the respective virus-specific amplicon of a different length. Five pairs of specific primers including PVY-6494F/PVY-7087R, ChiVMV-7987F/ChiVMV-8809R, TVBMV989F/TVBMV-2222R, TMV-130F/TMV-614R and CMV-265F/CMV-1240R were designed and shown in Table 1. The plasmid of each of the viruses tested, derived from previous studies, were used as positive 
controls. cDNAs were synthesized by reverse transcription of the total RNAs using HiScript $1^{\text {st }}$ Strand cDNA Synthesis Kit (Vazyme Biotech, Nanjing, China). The cDNAs were used as the templates (cigar tobacco leaves collected from Deyang with various disease symptoms are numbered as 1 to 16 and symptomatic flue-cured tobacco leaves collected from Luzhou are numbered as 17 to 22 , respectively). A series of 10 -fold dilutions from $1 \times 10^{8}$ to $1 \times 10^{2}$ copies of plasmid containing respective viral sequences was performed by using the templates to determine the lowest detection limit. PCR amplification were performed in $25 \mu \mathrm{L}$ final volume. Reaction components included $1 \mu \mathrm{L}$ of $10 \mu \mathrm{M}$ forward and reverse primers, $1 \mu \mathrm{L}$ cDNA and $12.5 \mu \mathrm{L}$ of $2 \times$ TaKaRa Premix (Ex Taq, $1.25 \mathrm{U} / 25 \mu \mathrm{L}$; dNTP Mixture, each 0.4 $\mathrm{mmol} / \mathrm{L} ; \mathrm{Mg}^{2+}, 4 \mathrm{mmol} / \mathrm{L}$ ) (Takara Bio, Dalian, China), and appropriate $\mathrm{ddH}_{2} \mathrm{O}$. The PCR procedures were as follows: pre-denaturation at $94^{\circ} \mathrm{C}$ for $5 \mathrm{~min} ; 30$ cycles of denaturation at $94^{\circ} \mathrm{C}$ for $30 \mathrm{~s}$, annealing at varying temperatures $55^{\circ} \mathrm{C}$ for $30 \mathrm{~s}$, and extension at $72^{\circ} \mathrm{C}$ for various durations $80 \mathrm{~s}$; and a final extension at $72^{\circ} \mathrm{C}$ for $10 \mathrm{~min}$. Finally, PCR products were separated and detected by $1.5 \%$ agarose gel electrophoresis and a gel imaging system (Bio-Rad, Hercules, CA, USA) was employed to observe the electrophoresis results.

\section{Results}

\section{Collection of samples with obvious symptoms on Tobacco and cigar tobacco in Sichuan province}

The climate in the Sichuan province is warm and humid with a large variety of plant viruses. In 2019, approximately 80 symptomatic leaf samples of cigar tobacco and flue-cured tobacco were collected from farm fields of Deyang and Luzhou city in Sichuan Province in China. The prevalent viral disease symptoms such as mosaic, venial necrosis, mottling, yellowing and necrotic spots were observed on leaves (Fig. 1). These samples were thereafter subjected to the sequencing analysis and virus identification.

\section{Identification of major viruses by High-throughput small RNA sequencing}

The small RNA libraries of two symptomatic cigar tobacco pooled samples from Deyang city and a symtomatic flue-cured tobacco pooled sample were constructed and sequenced on an Illumina HiSeq2000 platform which generated 28765749, 25093608 and 34935454 raw reads, respectively, and submitted to the Sequence Read Archive database at NCBI with the accession number SRR13499486, SRR13499485 and SRR13838825. Adapters and low quality reads were trimmed to obtain 27930450, 21537662 and 28194021 clean reads and the Q20 value were $99.01 \%, 98.86 \%$ and $99.07 \%$, respectively. The clean reads of each library were assembled into contigs through the homologydependent approach for plant virus identification. These contigs were searched against the nonredundant nucleotide sequence entries of the NCBI database by BLASTn. The results indicated that major viruses infecting flu-cured tobacco and cigar tobacco leaves in Deyang and Luzhou area were PVY, ChiVMV, TVBMV, TMV and CMV. Among witch, there were 27, 29, 2,14 and 33 contigs with the length range from 34 1720 nt that can be mapped to the genome sequences of PVY, ChiVMV, TVBMV, TMV and CMV (Fig. 2, Table S1). 


\section{Phylogenetic analysis}

Phylogenetic analysis was performed by MEGA7 based on the partial nucleotide sequences of PVY, ChiVMV, TVBMV, TMV and CMV collected from Deyang and Luzhou city in Sichuan province to investigate the evolutionary development of these viruses. The sequences of PVY-Deyang (MW419411.1) and PVY-Luzhou (MW419412.1) were compared to 15 other PVY isolates reported from various district as well as ChiVMV (JX088636.1) and TVBMV (NC-009994.1) as out groups were subjected to sequence alignment and phylogenetic analysis (Fig. 3A). The nucleotide sequences of PVY-Deyang and PVYLuzhou show high identity with $99.65 \%$. The sequence alignment also indicates that PVY-Deyang and PVY-Luzhou are most closely related with PVY-pt077-pot-18 (MN734254.1) on Solanum lycopersicum from Zhenjiang city of Jiangsu province China with $100.00 \%$ and $99.65 \%$ sequence identity, respectively. Results of the phylogenetic analysis also show that PVY-Deyang and PVY-Luzhou are distantly related with PVY-AST (JF928460.1) reported from Brazil and PVY-VNP413 (HG810950.1) reported from Viet Nam (Fig. 3A).

The sequences of ChiVMV-Deyang (MW355622.1) and ChiVMV-Luzhou (MK405594.1) as well as other 13 ChiVMV isolates together with PVY (KJ634023.1) and TVBMV (NC-009994.1) were subjected to sequence alignment and phylogenetic analysis (Fig. 3B). The results show that ChiVMV-Deyang and ChiVMV-Luzhou are most closely related with ChiVMV-Pp4 (KC711056) and Yp-8 (KC7110055) from pepper plants collected from Sichuan province with $99.42 \%$ and $99.03 \%$ sequence identity, respectively. Sequence of ChiVMV-Deyang also shows high sequence similarity with ChiVMV-Luzhou by $98.74 \%$. While both of the isolates are distantly related with ChiVMV-GD (KU987835.1), Wenchang (GQ981316.1) and Ca (AJ972878.1) isolates from Guandong, Hainan province in South China and South Korea (Fig. 3B).

The sequences of TVBMV-Deyang (MW419414.1) and TVBMV-Luzhou (MW419413.1) were compared with 14 other TVBMV isolates as well as the ChiVMV (JX088636.1) and PVY (KJ634023.1) to construct phylogenetic tree (Fig. 3C). TVBMV-Luzhou is closely related with TVBMV-XDB-04 (MG880245.1) and YN9.1(KF444434.1) from tobacco with $99.71 \%$ and $97.45 \%$ sequence identity, respectively. Interestingly, TVBMV-Deyang is most closely related with TVBMV-YN9.1 (KF444434.1) from tobacco with only $94.03 \%$ sequence identity and also shows comparatively lower sequence similarity with TVBMVLuzhou with $93.01 \%$ sequence identity. The results of phylogenetic analysis also indicate that TVBMVDeyang and TVBMV-Luzhou are distantly related with TVBMV SDWF11 (GU904096.1), YND (EF219408.1), JX (JN630471.1) isolates reported from Shandong province of China (Fig. 3C).

Phylogenetic analysis of TMV-Deyang (MW521350.1) and TMV-Luzhou (MW521351.1) were compared with other 18 reported TMV isolates together with a tomato mosaic virus (ToMV) (NC-002692.1) and a tobacco mild green mosaic virus (TMGMV) (NC-001566.1) isolate were used as the out groups (Fig. 3D). The results indicate that TMV-Deyang is closely related with Xiongfan-2 (HE818419.1) and Fumeng (HE818416.1) isolates with $99.75 \%$ and $98.95 \%$ sequence similarity. While TMV-Luzhou clustered with Xuyong (HE818454.1) as well as Tianzhu-1 (HE818444.1) isolate with $99.90 \%$ and $99.15 \%$ sequence similarity. The sequences identity of TMV-Deyang and TMV-Luzhou is $98.24 \%$ and both of the isolates 
are distantly related with Ohio V (FR878069.1), Harbin-2 (MH595920.1) and SXFQ (JX993906.1) (Fig. 3D).

The sequences of CMV-Deyang (MW698928.1) were compared to 20 other CMV isolates reported from various district as well as brome mosaic virus (NC-002028.2) and alfalfa mosaic virus (NC-002025.1) from USA as out groups and were subjected to sequence alignment and phylogenetic analysis (Fig. 3F). No CMV isolates were identified from Luzhou in this investigation. The results showed that CMV-Deyang is most closely related with CMV-RX9 (MH119149.1) and CMV-RX2 (MH119146.1) from Glycine max plants collected from China with $98.93 \%$ and $98.78 \%$ sequence identity, respectively, while distantly related with Ug90-RNA3 (MG021456.2), CMV-HC-56 (FM999062.1) and CMV-Jol186 (JX025994.1) isolates from Uganda, Thailand and Iran (Fig. 3F).

\section{Specificity, sensitivity and application of multiplex RT-PCR}

To simultaneously detect these viruses, we designed specific amplification primers for PVY, ChiVMV, TVBMV, TMV as well as CMV and constructed a multiplex RT-PCR detection system. To determine the sensitivity, a serious of plasmids including pEASY-PVY, pEASY-ChiVMV, pEASY-TVBMV, pCB-TMV-SN (no. MG516107) and pEASY-CMV were diluted from $10^{8}$ copies to $10^{2}$ copies, respectively, and amplified by the multiplex RT-PCR using $25 \mu \mathrm{L}$ reaction system. The results indicated that the detection limit of PVY, ChiVMV, TVBMV, TMV and CMV were $10^{4}, 10^{4}, 10^{4}, 10^{4}$ and $10^{5}$ copies, respectively (Fig. 4 A-E).

Furthermore, we mixed these plasmids at equal ratio and diluted from $10^{8}$ copies to $10^{2}$ copies each, and subjected to multiplex RT-PCR analysis. The results showed that 5 virus specific bands can generally be amplified from the mixed templates from $10^{8}$ to $10^{4}$ copies each (Fig. $4 \mathrm{~F}$ ), thereby indicating that the developed multiplex RT-PCR was sensitive and specific for simultaneous detection of PVY, ChiVMV, TVBMV, TMV and CMV.

To test the feasibility of the multiplex RT-PCR for simultaneous detection of these viruses in the field. We tested 22 field-collected symptomatic tobacco leaf samples from Deyang and Luzhou tobacco planting areas (Fig. 5A and B). The number of the leaf samples showed in Fig 5 A were consistent with lane number showed in Fig $5 \mathrm{~B}$. The results revealed that detection rate for PVY, ChiVMV, TVBMV, TMV and CMV were approximately $36 \%, 55 \%, 45 \%, 55 \%$ and $9 \%$, respectively. Among which, leaf samples (Fig $5 \mathrm{~A}$ and B. 1, 6, 7, 11 and 21) were infected by TMV, CMV, ChiVMV, TMV and ChiVMV, respectively. However, the other 17 leaf samples showed synergistic infection by two or more viruses. For example, two samples (Fig 5 A and B. 3 and 8) were synergistically infected by TVBMV and PVY. Leaf samples (Fig 5 A and B. 16, 20 and 22) indicated synergistic infection of ChiVMV and TMV. Additionally, leaf sample number 2 and 5 (TVBMV, ChiVMV and TMV), 9 (TVBMV, PVY and TMV), 10 (ChiVMV, PVY and TMV) and 18 (TVBMV, ChiVMV and PVY) were synergistically infected by three viruses (Fig. $5 \mathrm{~A}$ and B).

\section{Discussion}


In China, plant viruses cause extensive economic losses in tobacco production in spite of efforts on different control strategies (Tian et al., 2011). In addition to TMV, viruses belong to the potyvirus genus have become the major viral pathogens infecting tobacco (Akinyemi et al.,2016; Jiao et al., 2020). Sichuan province is one of the main tobacco production area in China (Jiao et al., 2020), where the tobacco plantation is usually surrounded or mixed with other crops, especially pepper plants, which increases the risk of multiple virus synergistic infection. Therefore, it is of great significance to accurately identify the species of plant viruses and establish specific and sensitive detection techniques for virus control.

To clarify the classification and the genetic relationship of the major viruses infecting tobacco plants in Deyang and Luzhou, we amplified partial sequences of three potyviruses including PVY, ChiVMV and TVBMV and subjected to nucleotide BLAST and phylogenetic analysis. The results indicated that PVYDeyang and PVY-Luzhou showed high sequence identity with each other, and also closely related with the isolates reported form Henan, Shandong, Heilongjiang and Hainan province in tobacco. We also compared amplified sequences of PVY Vpg and the contig sequences generated from RNA-seq with sequences of $P V Y^{N}, P V Y^{\circ}$ as well as $P V Y^{N T N}$. These results indicated high sequence similarity with $P V Y^{\circ}$ (data not shown), which suggested that the major PVY infecting tobacco plants in Sichuan province possibly be 0 strain.

Nucleotide BLAST and phylogenetic analysis suggested that TVBMV-Luzhou showed high similarity with TVBMV isolates previously reported form Shandong, Henan or Sichuan province, but comparably distantly related with that collected from Deyang in cigar tobacco. Interestingly, the sequence similarity between TVBMV-Deyang and the closest reported TVBMV isolate was only $94 \%$. It is possible that mutations or recombination have occurred in the evolution process of TVMBV-Deyang, which required more sampling and precise sequencing analysis of TVBMV to verify this possibility in the future study.

BLAST and phylogenetic analysis using the sequences of P3 of ChiVMV-Deyang or ChiVMV-Luzhou indicated that these isolates showed highest similarity with ChiVMV isolates collected from pepper plants in Sichuan province. This results indicated that the ChiVMV infected tobacco in Deyang and Luzhou should be originated from diseased pepper plants, which were in agreement with our previous study (Jiao et al., 2020). Synergistic infection of plant viruses can induce more severe disease symptoms on plants, which may cause greater losses and increase in difficulty for virus control. In this investigation, synergistic infection of ChiVMV with TVBMV, TMV or PVY can induce severe necrotic spots (Fig. 4 A-18; Fig. 4 B lane 18), which can be extensively observed in Luzhou tobacco planting area.

In this work, we designed specific amplification primers and established a sensitive and specific multiplex RT-PCR system for simultaneous detection of five major tobacco RNA viruses including PVY, ChiVMV, TVBMV, TMV and CMV, which is well applicate in tobacco growing areas in Sichuan province. Primer design is critical for the establishment of a practical multiplex RT-PCR detection system that requires the optimization of the specificity and sensitivity of the primers, and the distinguishable sizes of amplicon. In a previous study, a multiplex RT-PCR for the detection and classification of different PVY strains from 
potato including $\mathrm{PVY}^{\mathrm{N}}, \mathrm{PVY}^{\mathrm{O}}, \mathrm{PVY}^{\mathrm{NTN}}, \mathrm{PVY}^{\mathrm{NW}}$ and $\mathrm{PVY}^{\mathrm{NTN}-\mathrm{NW}}$ were developed based on different combination of amplicons (Chikh Ali et al., 2010). However, we used these primers to test our samples, but failed to amplify specific amplicons, which indicated that this reported multiplex RT-PCR analysis system may not be applicable in the classification of the precise PVY species that infect tobacco. A previous study has established a multiplex RT-PCR analysis for simultaneous detection of five plant virus infecting tobacco including PVY, TVBMV, TMV, TEV and CMV (Dai et al., 2012), but not including the detection of ChiVMV. In this work, we designed specific amplification primers based on the results of NGS and Sanger sequencing analysis and established a multiplex RT-PCR analysis, which is more suitable for the simultaneous detection of viruses in Sichuan tobacco planting area. Collectively, the developed multiplex RT-PCR assay was proved to be a sensitive diagnostic tool for the detection of the viruses from the leaves of naturally infected tobacco plants.

\section{Conclusion}

In this study, we identified three kinds of potyvirus including PVY, ChiVMV and TVBMV together with TMV as well as CMV from tobacco in the field of Deyang and Luzhou city in Sichuan province using next generation sequencing of small RNAs. A multiplex RT-PCR assay was developed as an easy, fast and sensitive method for the simultaneous detection of these frequent viruses infecting cigar and flue-cured tobacco crops in Sichuan. The results of this work also provide prompt valuable disease status information for tobacco breeders.

\section{Declarations}

\section{Funding}

This research was funded by National Key R\&D Program of China (2017YFD0201104) and Natural Science Foundation of China (32072391).

\section{Conflict of interest}

The authors declare that they have no confict of interest.

\section{Availability of data and material}

All data generated or analysed during this study are included in this published article and its supplementary information files.

\section{Code availability}

Not applicable

\section{Authors' contributions}


Conceptualization, Y.W., X.Z. and M.A.; methodology, T.Z.; software, T.Z. and M.A.; validation, T.Z.,Y.W., X.Z., Z.X. and M.A.; formal analysis, T.Z. and M.A.; investigation, Y.C., J.W., R.Z. and H.X.; resources, Y.W., X.Z. and M.A.; data curation, T.Z. and S.Z.; writing-original draft preparation, T.Z. and M.A.; writingreview and editing, T.Z. and Z.X.; visualization, Y.C., J.W., R.Z. and H.X.; supervision, Y.C., J.W., R.Z. and H.X.; project administration, Y.W., X.Z. and M.A. All authors have read and agreed to the published version of the manuscript.

\section{Ethics approval}

Ethical approval not required. Standard ethical and professional conduct have been followed.

\section{Consent to participate}

All participants were informed and agreed.

\section{Consent for publication}

All participants agreed to publish.

\section{References}

1. Akinyemi IA, Wang F, Zhou B, Qi S, Wu Q (2016) Ecogenomic survey of plant viruses infecting tobacco by next generation sequencing. Virology J. 13,181

2. Bi X, Li X, Yu H, An M, Li R, Xia Z, Wu Y (2019) Development of a multiplex RT-PCR assay for simultaneous detection of Cucumber green mottle mosaic virus and Acidovorax citrulli in watermelon. PeerJ. 7,e7539

3. Chikh Ali M, Maoka T, Natsuaki KT, Natsuaki T (2010) The simultaneous differentiation of Potato virus $Y$ strains including the newly described strain PVY(NTN-NW) by multiplex PCR assay. J Virol Methods 165:15-20

4. Cuevas JM, Delaunay A, Visser JC, Bellstedt DU, Jacquot E, Elena SF (2012) Phylogeography and molecular evolution of potato virus Y. PLoS One 7:e37853

5. Dai J, Cheng J, Huang T, Zheng X, Wu Y (2012) A multiplex reverse transcription PCR assay for simultaneous detection of five tobacco viruses in tobacco plants. J Virol Methods 183:57-62

6. Ding SW, Lu R (2011) Virus-derived siRNAs and piRNAs in immunity and pathogenesis. Curr Opin Virol 1:533-544

7. Faurez F, Baldwin T, Tribodet M, Jacquot E (2012) Identification of new Potato virus Y (PVY) molecular determinants for the induction of vein necrosis in tobacco. Mol Plant Pathol 13:948-959

8. Geng C, Wang HY, Liu J, Yan ZY, Tian YP, Yuan XF, Gao R, Li XD (2017) Transcriptomic changes in Nicotiana benthamiana plants inoculated with the wild-type or an attenuated mutant of Tobacco vein banding mosaic virus. Mol Plant Pathol 18:1175-1188 
9. Jiao Y, Xu C, Li J, Gu Y, Xia C, Xie Q, Xie Y, An M, Xia Z, Wu Y (2020) Characterization and a RT-RPA assay for rapid detection of Chilli Veinal mottle virus (ChiVMV) in tobacco. Virol J 17:33

10. Kreuze JF, Perez A, Untiveros M, Quispe D, Fuentes S, Barker I, Simon R (2009) Complete viral genome sequence and discovery of novel viruses by deep sequencing of small RNAs: a generic method for diagnosis, discovery and sequencing of viruses. Virology 388:1-7

11. Kumar S, Stecher G, Tamura K (2016) MEGA7: Molecular Evolutionary Genetics Analysis Version 7.0 for bigger datasets. Mol Biol Evol 33:1870-1874

12. Moreno AB, López-Moya JJ (2020) When viruses play team sports: Mixed infections in plants. Phytopathology 110:29-48

13. Rao S, Chen X, Qiu S, Peng J, Zheng H, Lu Y, Wu G, Chen J, Jiang W, Zhang Y, Yan F (2020) Identification of two new isolates of chilli veinal mottle virus from different regions in China: Molecular diversity, phylogenetic and recombination analysis. Front Microbiol 11:616171

14. Scholthof KB, Adkins S, Czosnek H, Palukaitis P, Jacquot E, Hohn T, Hohn B, Saunders K, Candresse T, Ahlquist P, Hemenway C, Foster G (2011) Top 10 plant viruses in molecular plant pathology. Mol Plant Pathol 12:938-954

15. Shaw JG (1999) Tobacco mosaic virus and the study of early events in virus infections. Philos Trans R Soc Lond B Biol Sci 354:603-611

16. Su CL, Chao YT, Alex Chang YC, Chen WC, Chen CY, Lee AY, Hwa KT, Shih MC (2011) De novo assembly of expressed transcripts and global analysis of the Phalaenopsis aphrodite transcriptome. Plant Cell Physiol 52:1501-1514

17. Sun HJ, Liu W, Yang JG, Wang FG, Shen LL, Li Y, Sun DM, Qian YM (2016) Complete genome sequence of a novel wild tomato mosaic virus isolate infecting Nicotiana tabacum in China. J Phytopathol. 164,686

18. Tian YP, Liu JL, Zhang CL, Liu YY, Wang B, Li XD, Guo ZK, Valkonen JP (2011) Genetic diversity of potato virus $Y$ infecting tobacco crops in China. Phytopathology 101:377-387

19. Tian YP, Valkonen JP (2015) Recombination of strain O segments to HCpro-encoding sequence of strain $\mathrm{N}$ of Potato virus $\mathrm{Y}$ modulates necrosis induced in tobacco and in potatoes carrying resistance genes Ny or Nc. Mol Plant Pathol 16:735-747

20. Vivek AT, Zahra S, Kumar S (2020) From current knowledge to best practice: A primer on viral diagnostics using deep se-quencing of virus-derived small interfering RNAs (vsiRNAs) in infected plants. Methods 183:30-37

21. Wang C, Wang C, Zou J, Yang Y, Li Z, Zhu S (2019) Epigenetics in the plant-virus interaction. Plant Cell Rep 38:1031-1038

22. Wu Q, Wang Y, Cao M, Pantaleo V, Burgyan J, Li WX, Ding SW (2012) Homology-independent discovery of replicating pathogenic circular RNAs by deep sequencing and a new computational algorithm. Proc Natl Acad Sci U S A 109:3938-3943

23. Xia Z, Zhao Z, Chen L, Li M, Zhou T, Deng C, Zhou Q, Fan Z (2016) Synergistic infection of two viruses MCMV and SCMV increases the accumulations of both MCMV and MCMV-derived siRNAs in 
maize. Sci Rep 6:20520

24. Yang T, Xu ZP, Lv R, Zhu LS, Peng QD, Qiu L, Lin HH, Xi DH (2018) N gene enhances resistance to Chilli veinal mottle virus and hypersensitivity to salt stress in tobacco. J Plant Physiol 230:92-100

25. Zhang C, Wu Z, Li Y, Wu J (2015) Biogenesis, function, and applications of virus-derived small RNAs in plants. Front Microbiol 6:1237

26. Zhou B, Deng YM, Barnes JR, Sessions OM, Chou TW, Wilson M, Stark TJ, Volk M, Spirason N, Halpin RA (2017) Multiplex Reverse Transcription-PCR for Simultaneous Surveillance of Influenza A and B Viruses. J Clin Microbiol 55:3492-3501

\section{Tables}

Table 1. Primer list used for phylogenetic analysis and multiplex RT-PCR 


\begin{tabular}{|c|c|c|}
\hline primer name & sequence & amplification size \\
\hline PVY-5719F & GGGAAAAATAAATCCAAAAG & \multirow[t]{2}{*}{564 bp } \\
\hline PVY-6263R & TTCATGCTCCACTTCCTGTT & \\
\hline ChiVMV-2441F & GGTGAAGGAATTGAAAGTAT & \multirow[t]{2}{*}{1032 bp } \\
\hline ChiVMV-3453R & TTGATGACCCACTGGTTCCT & \\
\hline TVBMV-1071F & TCAGCAGCAGAACAATTCTG & \multirow[t]{2}{*}{1374 bp } \\
\hline TVBMV-2425R & ACCAACTCTATAATGCTTCA & \\
\hline TMV-70F & ATGGCATACACACAGACAGC & \multirow[t]{2}{*}{1992 bp } \\
\hline TMV-2061R & CTCCGGATGATCTCCAGCAA & \\
\hline CMV -1257F & ATGGACAAATCTGAATCAAC & \multirow[t]{2}{*}{657 bp } \\
\hline CMV-1913R & CAGGGGTGTTCCCAGTTTGA & \\
\hline PVY-6494F & GATGGGCATTGTGGATTACC & \multirow[t]{2}{*}{613 bp } \\
\hline PVY-7087R & TCTGGAAGCCATGCACTTGC & \\
\hline ChiVMV-7987F & AATGGCAATGCGGTATTCAC & \multirow[t]{2}{*}{842 bp } \\
\hline ChiVMV-8809R & CGACGGCCTTCGTCTTAACC & \\
\hline TVBMV-989F & TGTGCGAGGTCGTGATGGGG & \multirow[t]{2}{*}{$1253 \mathrm{bp}$} \\
\hline TVBMV-2222R & GATAGCATGCAAGTGCAACC & \\
\hline TMV-130F & TCCTTGGTCAATGATCTAGC & \multirow[t]{2}{*}{485 bp } \\
\hline TMV-614R & GTATTGTGACAGACAGCGTC & \\
\hline CMV-265F & TTCCGAGGTCATCCGGAATC & \multirow[t]{2}{*}{995 bp } \\
\hline CMV-1240R & CCTTGAACTGTTCCATCCAC & \\
\hline
\end{tabular}

\section{Figures}




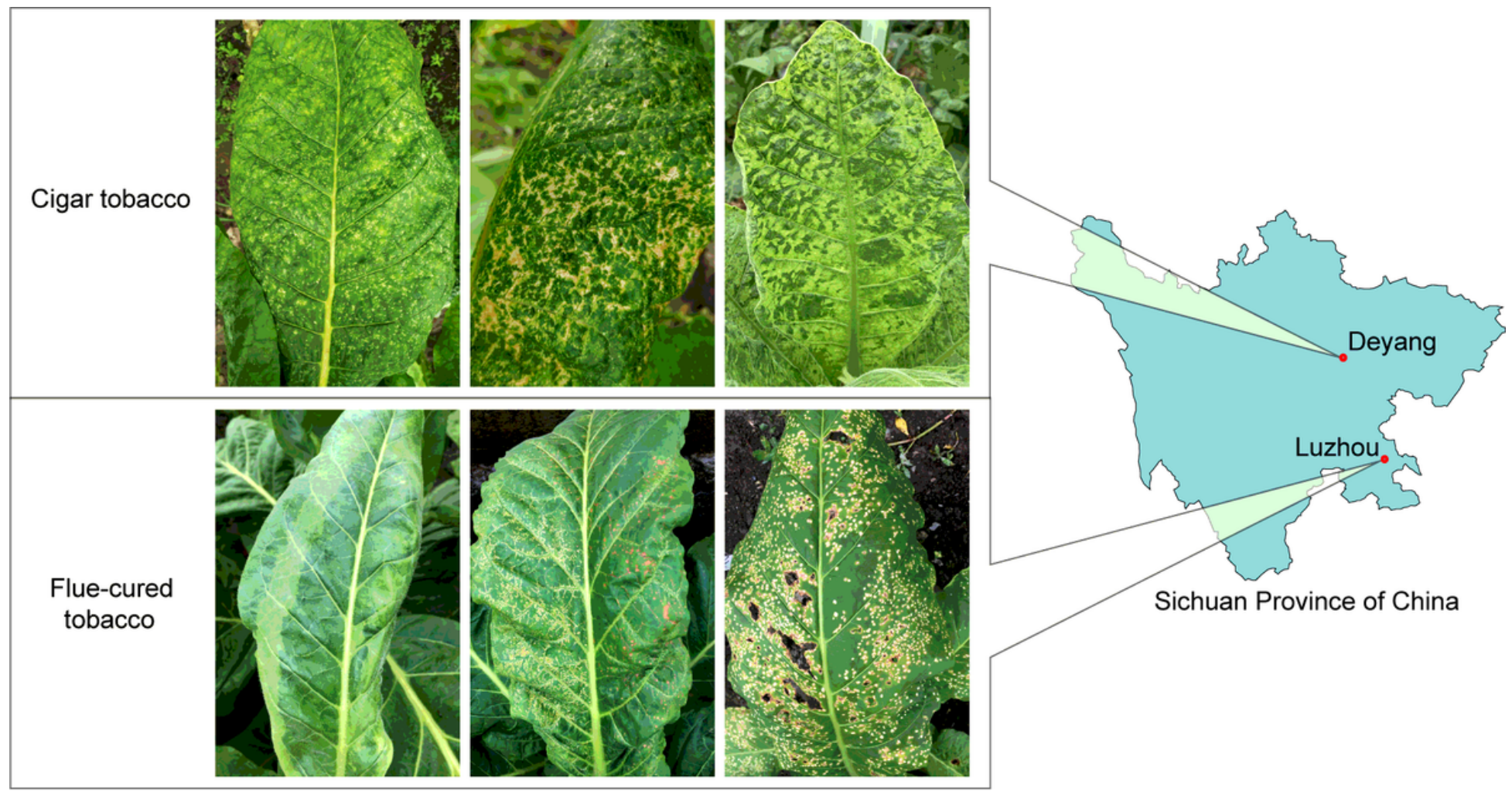

Figure 1

Cigar and flue-cured tobacco leaf samples with typical virus disease symptoms were collected from Deyang and Luzhou city in Sichuan Province of China. 


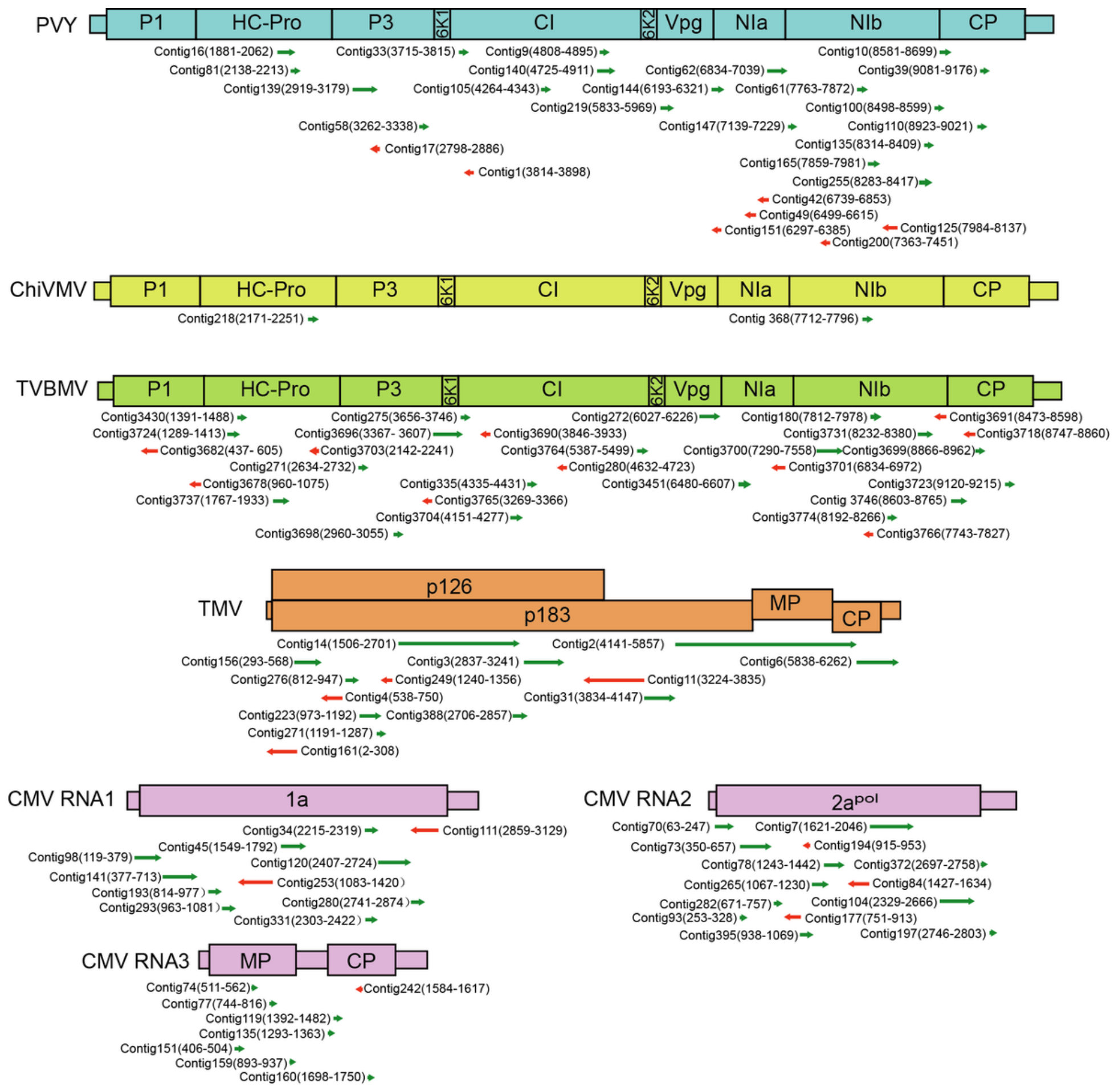

\section{Figure 2}

Contigs mapped to the genomes of PVY, ChiVMV, TVBMV, TMV and CMV, respectively, based on the results of high-throughput small RNA sequencing. The contigs mapped to the virus positive-strand RNA were shown in green color and those mapped to negative-strand RNA were shown in red color. 
A

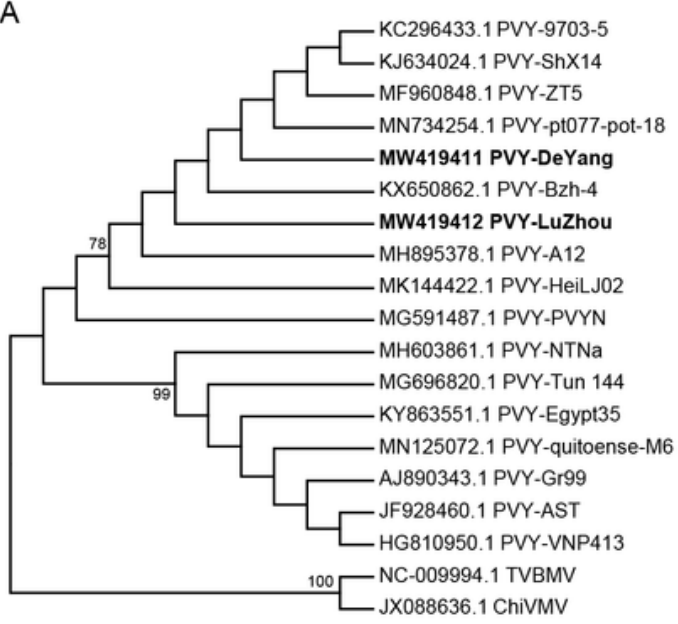

C

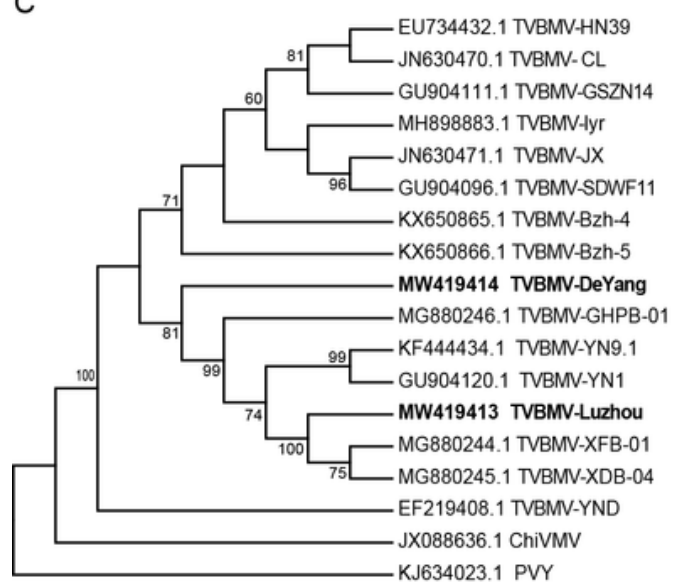

B
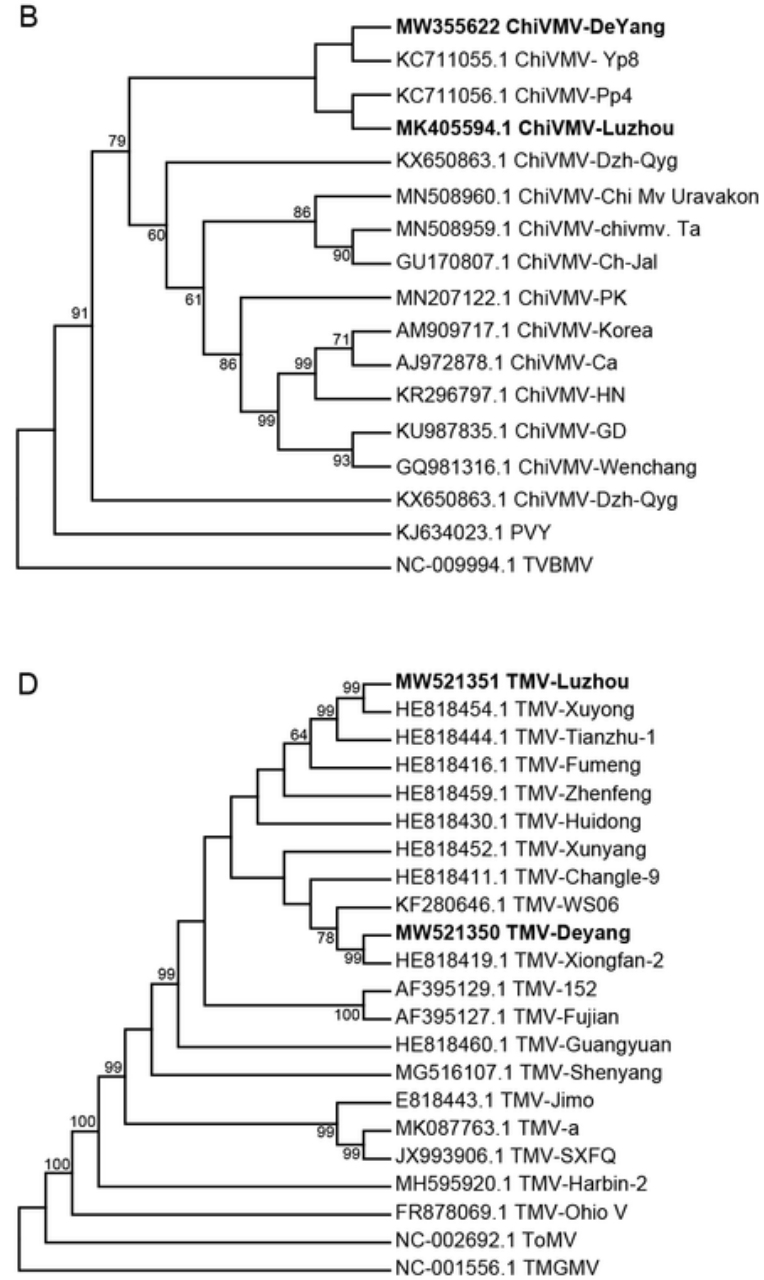

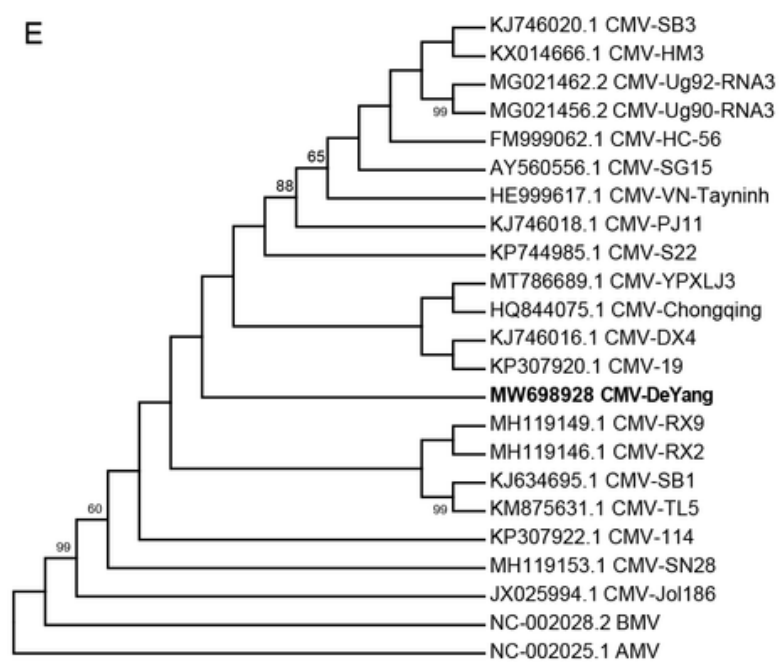

Figure 3

Phylogenetic analysis performed by MEGA7 based on the nucleotide sequences of five identified plant viruses collected from Deyang and Luzhou. (A) Phylogenetic tree of PVY. (B) Phylogenetic tree of ChiVMV. (C) Phylogenetic tree of TVBMV. (D) Phylogenetic tree of TMV. (E) Phylogenetic tree of CMV. The analysis was performed using Maximum Likelihood method and numbers at the nodes of the branches represent percentage bootstrap values (1000 replicates). 

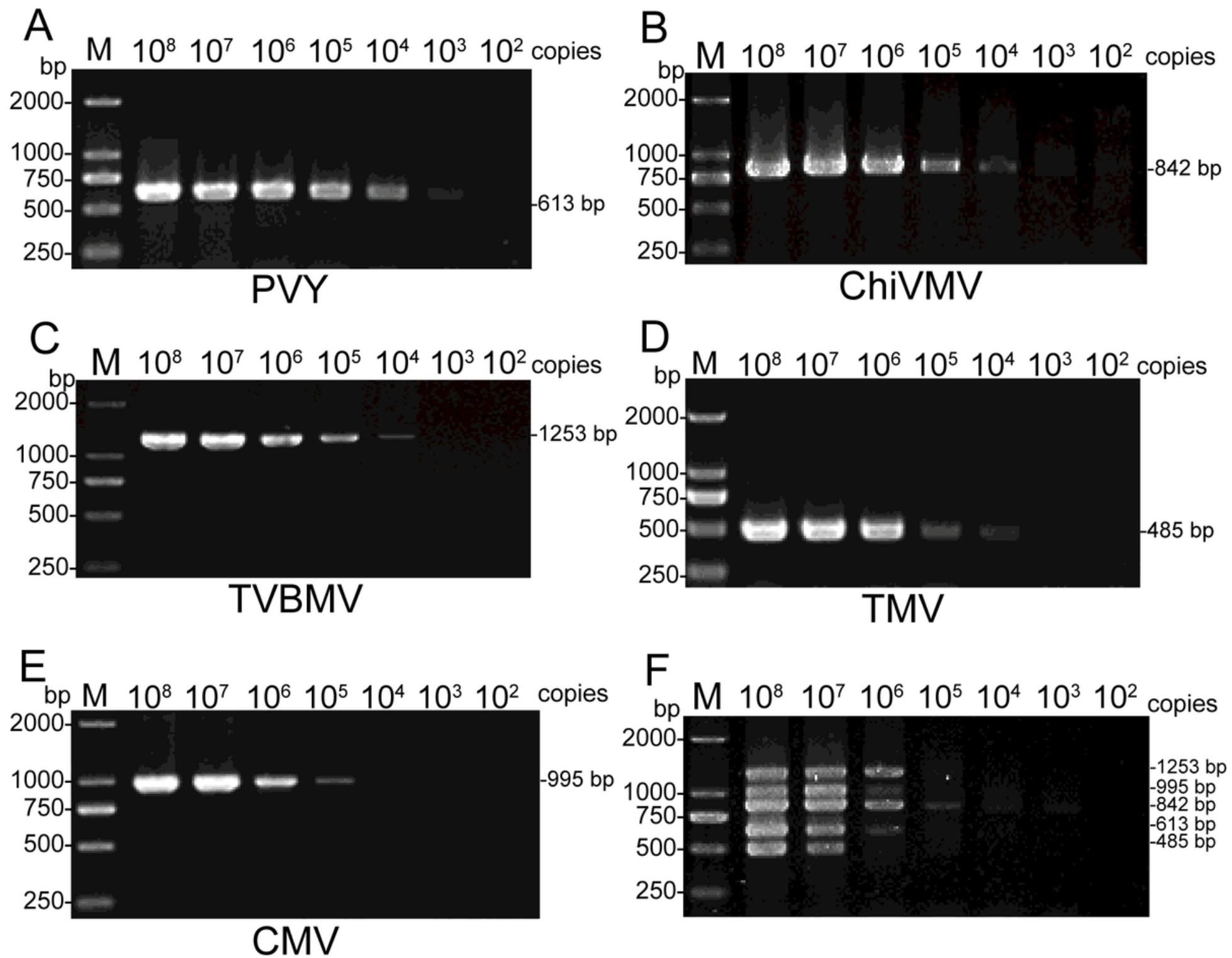

Figure 4

Multiplex RT-PCR detection of tobacco-infecting PVY, ChiVMV, TVBMV, TMV and CMV, and its application in field sample detection. (A-E) Sensitivity and specificity of multiplex RT-PCR were tested by detecting the plasmids containing virus sequences (pEASY-PVY, pEASY-ChiVMV, pEASY-TVBMV, pCB-TMV-SY and pEASY-CMV, respectively) diluted from $1 \times 108$ to $1 \times 102$ copies, each. $(F)$. Simultaneous detection of these mixed plasmids diluted from $1 \times 108$ to $1 \times 102$ copies using multiplex RT-PCR.

原

\section{Figure 5}

Disease diagnosis of field-grown cigar tobacco and flue-cured tobacco leaf samples by multiplex RT-PCR. (A)Typical yellow mosaic, deformation, stunting and necrotic spot symptoms of the tobacco samples collected from Deyang (cigar tobacco leaf sample 1-17) and Luzhou (flue-cured tobacco leaf sample 1822) city of Sichuan Province. (B) Detection of PVY, ChiVMV, TVBMV TMV, and CMV from leaves of 
tobacco using multiplex RT-PCR. Lanes 1-16, leaf samples of cigar tobacco from Deyang city. 17-22, leaf samples of flue-cured tobacco from Luzhou city. Lane 23 is a negative control.

\section{Supplementary Files}

This is a list of supplementary files associated with this preprint. Click to download.

- TableS1.xIsx 